\title{
On Isospectral Sets of Jacobi Operators
}

\author{
F. Gesztesy ${ }^{1, *}$, M. Krishna ${ }^{2, * *}$, G. Teschl ${ }^{1, * * *}$ \\ ${ }^{1}$ Department of Mathematics, University of Missouri, Columbia, MO 65211, USA \\ 2 Institute of Mathematical Sciences, Taramani, Madras 600113 , India
}

Received: 9 January 1996/Accepted: 16 April 1996

\begin{abstract}
We consider the inverse spectral problem for a class of reflectionless bounded Jacobi operators with empty singularly continuous spectra. Our spectral hypotheses admit countably many accumulation points in the set of eigenvalues as well as in the set of boundary points of intervals of absolutely continuous spectrum. The corresponding isospectral set of Jacobi operators is explicitly determined in terms of Dirichlet-type data.
\end{abstract}

\section{Introduction}

The principal aim of this paper is to study certain bounded self-adjoint Jacobi operators whose inverse spectral theory and isospectral class can be characterized explicitly.

Since the literature on inverse spectral theory for Jacobi operators (especially in the periodic and short-range scattering case) is rather extensive, we confine ourselves to a brief account of those results which are close in spirit to our approach. In this context, the use of auxiliary spectral problems of the Dirichlet-type in connection with either the moment problem or the algebro-geometric approach to (quasi-)periodic finite-gap Jacobi operators, comes to mind first. Dirichlet spectra and the moment problem were first combined in the pioneering work by Kac and van Moerbeke [23, 24, 29]. The Jacobi inversion problem in connection with Dirichlet divisors appeared in Date and Tanaka [12] (see also [36]) and simultaneously in Dubrovin, Matveev, and Novikov [15] with further developments in $[28,30,31]$. (The algebro-geometric method is presented in great detail in [6].) A complete algebro-geometric treatment of Toda and Kac-van Moerbeke hierarchies can be found in [8]; the isospectral torus of quasi-periodic Jacobi operators is explicitly realized in [20]. The next step involved extensions to certain almost

\footnotetext{
* E-mail: mathfg@mizzou1.missouri.edu

** E-mail: krishna@imsc.ernet.in

*** E-mail: teschl@iram.rwth-aachen.de
} 
periodic and random stationary Jacobi operators with infinitely many gaps in their spectrum. Based on fundamental contributions by Levitan [26], followed by Kotani and Krishna [25] and Craig [11] in the case of Schrödinger operators, Antony and Krishna [2, 3] and especially Sodin and Yuditskii $[34,35]$ characterized the inverse spectral problem for certain classes of almost periodic Jacobi operators by solving an infinite dimensional Jacobi inversion problem. In the random case Carmona and Kotani [9] provided necessary and sufficient conditions for a Herglotz function to be the expectation of a half-line Weyl $m$-function for a class of random stationary Jacobi operators. These extensions use elements of harmonic analysis, in particular, Herglotz properties of diagonal Green's functions and their boundary behavior on the real line.

In this paper we consider a different class of bounded Jacobi operators $H$ with infinitely many gaps in their spectrum. More precisely, we assume that the spectrum $\Sigma$ of $H$ satisfies

$$
\Sigma=\mathbb{R} \backslash \bigcup_{j \in J_{0} \cup\{\infty\}} \rho_{j},
$$

where $J \subseteq \mathbb{N}, J_{0}=J \cup\{0\}$,

$$
\begin{aligned}
\rho_{0}=\left(-\infty, E_{0}\right), & \rho_{\infty}=\left(E_{\infty}, \infty\right), \\
E_{0} \leqq E_{2 j-1}<E_{2 j} \leqq E_{\infty}, & \rho_{j}=\left(E_{2 j-1}, E_{2 j}\right), \quad j \in J, \\
-\infty<E_{0}<E_{\infty}<\infty, & \rho_{j} \cap \rho_{k}=\emptyset \text { for } j \neq k
\end{aligned}
$$

such that the set $\mathscr{A}$ of all accumulation points of $\left\{E_{2 j-1}, E_{2 j}\right\}_{j \in J}$ is countable, that is,

$$
\mathscr{A}=\left\{E_{2 j-1}, E_{2 j}\right\}_{j \in J}^{\prime} \quad \text { is countable . }
$$

(Here $A^{\prime}$ denotes the derived set of $A \subset \mathbb{R}$, i.e., the set of all accumulation points of $A$.)

Hypotheses (1.1)-(1.3) include situations such as the class of algebro-geometric finite-gap Jacobi operators on one hand and Jacobi operators with pure point spectrum with at most countably many accumulation points on the other hand. Our methods integrate the use of trace formulas and Herglotz functions (as recently outlined by Gesztesy and Simon [17, 18]) and the moment problem. In particular, the isospectral set of all Jacobi operators with spectrum $\Sigma$ satisfying (1.1)-(1.3) is explicitly determined in Theorem 4.3.

\section{Preliminaries}

In this section we recall some of the basic facts on Jacobi operators needed in Sects. 3 and 4. Detailed accounts of this material can be found, for instance, in [7], Ch. VII, [10], Ch. III, [20], Appendices A-D.

Let $\{a(m)>0\}_{m \in \mathbb{Z}},\{b(m)\}_{m \in \mathbb{Z}} \in \ell_{\mathbb{R}}^{\infty}(\mathbb{Z})$ be bounded real-valued sequences and introduce the bounded self-adjoint Jacobi operator $H$ in $\ell^{2}(\mathbb{Z})$ by

$$
(H f)(m)=(\tau f)(m), \quad f=\{f(m)\}_{m \in \mathbb{Z}} \in \ell^{2}(\mathbb{Z}),
$$

with the difference expression $\tau$ defined by

$$
(\tau f)(m)=a(m) f(m+1)+a(m-1) f(m-1)+b(m) f(m), \quad m \in \mathbb{Z} .
$$


The Green's function $G(z, m, n)$ associated with the resolvent $(H-z)^{-1}$ of $H$ then can be represented by

$$
\begin{aligned}
G\left(z, n, n^{\prime}\right)= & \left(\delta(n),(H-z)^{-1} \delta\left(n^{\prime}\right)\right) \\
= & W\left(u_{-}(z), u_{+}(z)\right)^{-1}\left\{\begin{array}{ll}
u_{-}(z, n) u_{+}\left(z, n^{\prime}\right), & n \leqq n^{\prime} \\
u_{+}(z, n) u_{-}\left(z, n^{\prime}\right), & n \geqq n^{\prime}
\end{array},\right. \\
z \in \mathbb{C} \backslash \sigma(H), & n, n^{\prime} \in \mathbb{Z} .
\end{aligned}
$$

Here $\delta(n)=\left\{\delta_{m, n}\right\}_{m \in \mathbb{Z}}, \sigma(\cdot)$ abbreviates the spectrum, $u_{ \pm}(z, \cdot)$ are Weyl solutions satisfying

$$
\tau u_{ \pm}(z)=z u_{ \pm}(z), \quad u_{ \pm}(z, \cdot) \in \ell^{2}\left(\left(m_{0}, \pm \infty\right) \cap \mathbb{Z}\right), m_{0} \in \mathbb{Z}, z \in \mathbb{C} \backslash(H),
$$

and $W(f, g)(n)$ denotes the Wronskian

$$
W(f, g)(m)=a(m)[f(m) g(m+1)-f(m+1) g(m)], \quad m \in \mathbb{Z} .
$$

Since $H$ is in the limit point case at $\pm \infty, u_{ \pm}(z, \cdot)$ are unique up to constant multiples. They can be chosen to be holomorphic for $z \in \mathbb{C} \backslash \sigma_{\text {ess }}(H)\left(\sigma_{\text {ess }}(\cdot)\right.$ denoting the essential spectrum).

Next, denote by $H_{ \pm, n}, n \in \mathbb{Z}$ the restrictions of $H$ to $\ell^{2}([n \pm 1, \pm \infty) \cap \mathbb{Z})$ with a Dirichlet boundary condition at the point $n \in \mathbb{Z}$, that is,

$$
\left(H_{ \pm, n} f\right)(m)=(\tau f)(m), \quad f \in\left\{g \in \ell^{2}([n \pm 1, \pm \infty) \cap \mathbb{Z}) \mid g(n)=0\right\} .
$$

The Weyl $m$-functions $m_{ \pm, n}(z)$ associated with $H_{ \pm, n}$ are then given by

$$
\begin{aligned}
m_{ \pm, n}(z) & =\left(\delta(n \pm 1),\left(H_{ \pm, n}-z\right)^{-1} \delta(n \pm 1)\right) \\
& =\left\{\begin{array}{l}
-u_{+}(z, n+1) /\left[a(n) u_{+}(z, n)\right] \\
-u_{-}(z, n-1) /\left[a(n-1) u_{-}(z, n)\right]
\end{array} .\right.
\end{aligned}
$$

Introducing the abbreviations,

$$
\begin{aligned}
g(z, n) & =G(z, n, n), \\
h(z, n) & =2 a(n) G(z, n, n+1)-1, \\
M_{+}(z, n) & =a(n)^{2} m_{+, n}(z), \\
M_{-}(z, n) & =a(n-1)^{2} m_{-, n}(z)+z-b(n),
\end{aligned}
$$

one infers

$$
\begin{gathered}
g(z, n)=-\left[M_{+}(z, n)+M_{-}(z, n)\right]^{-1}, \\
a(n)^{2} g(z, n+1)=M_{+}(z, n) M_{-}(z, n)\left[M_{+}(z, n)+M_{-}(z, n)\right]^{-1}, \\
h(z, n)=\left[M_{+}(z, n)-M_{-}(z, n)\right]\left[M_{+}(z, n)+M_{-}(z, n)\right]^{-1} .
\end{gathered}
$$

We recall that for all $n \in \mathbb{Z}, g(\cdot, n)$ and $M_{ \pm}(\cdot, n)$ are Herglotz functions (in contrast to $h(\cdot, n))$. Finally, if $\sigma_{p}(\cdot)$ abbreviates the point spectrum (i.e., the set 
of eigenvalues) one obtains

$E \in \sigma_{p}(H)$ if and only if $-\lim _{\varepsilon \downarrow 0} i \varepsilon[g(E+i \varepsilon, n)+g(E+i \varepsilon, n+1)]>0$,

$$
\mu \in \sigma_{p}\left(H_{ \pm, n}\right) \text { if and only if }-\lim _{\varepsilon \downarrow 0} i \varepsilon M_{ \pm}(\mu+i \varepsilon, n)>0
$$

\section{The Direct Spectral Problem}

In this section we discuss the direct spectral problem for a certain class of reflectionless bounded Jacobi operators.

In order to set the stage we first recall that $g(z, n)$ admits an exponential Herglotz representation [5] of the form

$$
g(z, n)=|g(i, n)| \exp \left\{\int_{\mathbb{R}}\left[\frac{1}{\lambda-z}-\frac{\lambda}{1+\lambda^{2}}\right] \xi(\lambda, n) d \lambda\right\},
$$

where, for all $n \in \mathbb{Z}$,

$$
\begin{gathered}
0 \leqq \xi(\lambda, n) \leqq 1 \text { for a.e. } \lambda \in \mathbb{R}, \\
\xi(\lambda, n)=\lim _{\varepsilon \downarrow 0} \operatorname{Im}\{\ln [g(\lambda+i \varepsilon, n)]\} \quad \text { for a.e. } \lambda \in \mathbb{R} .
\end{gathered}
$$

One can normalize $\xi(\lambda, n)$ by demanding

$$
\xi(\lambda, n)=0 \text { for } \lambda<\inf \{\sigma(H)\} .
$$

Our principal spectral hypothesis on $H$ then reads as follows.

Hypothesis 3.1. (i) $H$ is a bounded self-adjoint Jacobi operator. Hence its spectrum can be written as

$$
\sigma(H)=\mathbb{R} \backslash \bigcup_{j \in J_{0} \cup\{\infty\}} \rho_{j},
$$

where $J \subseteq \mathbb{N}, J_{0}=J \cup\{0\}$,

$$
\begin{array}{rlrl}
\rho_{0}=\left(-\infty, E_{0}\right), & & \rho_{\infty}=\left(E_{\infty}, \infty\right), \\
E_{0} \leqq E_{2 j-1}<E_{2 j} \leqq E_{\infty}, & & \rho_{j}=\left(E_{2 j-1}, E_{2 j}\right), \quad j \in J, \\
-\infty<E_{0}<E_{\infty}<\infty, & \rho_{j} \cap \rho_{k}=\emptyset \quad \text { for } j \neq k .
\end{array}
$$

(ii) The set of all accumulation points of the set $\left\{E_{2 j-1}, E_{2 j}\right\}_{j \in J}$ is assumed to be countable and denoted by

$$
\mathscr{A}=\left\{\tilde{E}_{j}\right\}_{j \in \tilde{J}}, \quad \tilde{J} \subseteq \mathbb{N} .
$$

(iii) For all $n \in \mathbb{Z}$,

$$
\xi(\lambda, n)=\frac{1}{2} \text { for a.e. } \lambda \in \sigma_{\mathrm{ess}}(H)
$$


We emphasize that the notation employed in (3.6) implies that $E_{2 \ell}=E_{2 k+1}$ for some $k \in J_{0} \cup\{\infty\}$ whenever $E_{2 l} \in \sigma_{d}(H)\left(\sigma_{d}(\cdot)\right.$ abbreviating the discrete spectrum).

Remark 3.2. (i) Since by hypotheses (3.5) and (3.6), $\sigma(H) \subset\left[E_{0}, E_{\infty}\right]$ is bounded, the corresponding sequences $\{a(n)>0\}_{n \in \mathbb{Z}}$ and $\{b(n)\}_{n \in \mathbb{Z}} \subset \mathbb{R}$ associated with the difference expression (2.2) are necessarily bounded.

(ii) Hypothesis (H.3.1) (i) implies that $\sigma(H)$ is a countable union of closed intervals (which may degenerate to points) of the type,

$$
\sigma(H)=\left(\bigcup_{j \in J_{0}} \Sigma_{j}\right) \cup\left(\bigcup_{j \in \tilde{J}} \tilde{\Sigma}_{j}\right),
$$

where

$$
\Sigma_{j}=\left[E_{2 j}, E_{2 j}^{(r)}\right], \quad j \in J_{0}, \quad \tilde{\Sigma}_{j}=\left[\tilde{E}_{j}, \tilde{E}_{j}^{(r)}\right], \quad j \in \tilde{J}
$$

with

$$
x^{(r)}=\inf \left\{E_{n} \in\left[E_{0}, E_{\infty}\right] \mid x<E_{n}\right\} \quad \text { for } x \in\left[E_{0}, E_{\infty}\right] .
$$

Reflectionless conditions such as (3.8) have been used by a variety of authors for particular cases such as almost periodic potentials (see, e.g., [2, 3, 11, 14, 25, $34,35]$ ) and scattering theoretic situations (cf., e.g., $[13,16])$. The following result further illustrates (H.3.1) (iii).

Lemma 3.3. Suppose $H$ is a bounded Jacobi operator and $\Omega \subset \sigma(H)$. Then the following conditions are equivalent:

(i) For all $n \in \mathbb{Z}, \xi(\lambda, n)=\frac{1}{2}$ for a.e. $\lambda \in \Omega$.

(ii) For some $n_{0} \in \mathbb{Z}, n_{1} \in \mathbb{Z} \backslash\left\{n_{0}, n_{0}+1\right\}$,

$$
\xi\left(\lambda, n_{0}\right)=\xi\left(\lambda, n_{0}+1\right)=\xi\left(\lambda, n_{1}\right)=\frac{1}{2} \quad \text { for a.e } \lambda \in \Omega .
$$

(iii) For some $n_{0} \in \mathbb{Z}$,

$$
M_{+}\left(\lambda+i 0, n_{0}\right)=-\overline{M_{-}\left(\lambda+i 0, n_{0}\right)} \text { for a.e. } \lambda \in \Omega .
$$

Proof. Clearly (i) implies (ii). In order to prove that (ii) implies (iii) we first recall that $M_{ \pm}\left(z, n_{0}\right)$, being Herglotz functions, have nontangential limits $z \rightarrow \lambda$ for a.e. $\lambda \in \mathbb{R}$. Next, consider a particular representation of $u_{ \pm}\left(z, n, n_{0}\right)$ in (2.7) normalized by $u_{ \pm}\left(z, n_{0}, n_{0}\right)=1, z \in \mathbb{C} \backslash \sigma\left(H_{ \pm, n_{0}}\right)$, given by

$$
u_{ \pm}\left(z, n, n_{0}\right)=c\left(z, n, n_{0}\right) \mp a\left(n_{0}\right)^{-1} M_{ \pm}\left(z, n_{0}\right) s\left(z, n, n_{0}\right) .
$$

Here $c\left(z, n, n_{0}\right), s\left(z, n, n_{0}\right)$ are solutions of $\tau \psi(z)=z \psi(z), z \in \mathbb{C}$ defined by

$$
s\left(z, n_{0}, n_{0}\right)=c\left(z, n_{0}+1, n_{0}\right)=0, \quad s\left(z, n_{0}+1, n_{0}\right)=c\left(z, n_{0}, n_{0}\right)=1,
$$

in particular, $c\left(z, n, n_{0}\right), s\left(z, n, n_{0}\right)$ are polynomials with respect to $z$ and real-valued for $z \in \mathbb{R}$. The requirement $\xi(\lambda, m)=\frac{1}{2}$, that is, $g(\lambda+i 0, m)=\overline{-g(\lambda+i 0, m)}$ for a.e. $\lambda \in \Omega$ then yields upon choosing $m=n_{0}$,

$$
\operatorname{Re}\left[M_{+}\left(\lambda+i 0, n_{0}\right)\right]=-\operatorname{Re}\left[M_{-}\left(\lambda+i 0, n_{0}\right)\right] \text { for a.e. } \lambda \in \Omega
$$


and

$$
\begin{aligned}
& {\left[M_{+}\left(\lambda+i 0, n_{0}\right) M_{-}\left(\lambda+i 0, n_{0}\right)-\overline{M_{+}\left(\lambda+i 0, n_{0}\right) M_{-}\left(\lambda+i 0, n_{0}\right)}\right] s\left(\lambda, m, n_{0}\right)^{2}} \\
& \quad-\left\{\operatorname{Im}\left[M_{+}\left(\lambda+i 0, n_{0}\right)\right]-\operatorname{Im}\left[M_{-}\left(\lambda+i 0, n_{0}\right)\right]\right\} 2 i a\left(n_{0}\right) c\left(\lambda, m, n_{0}\right) s\left(\lambda, m, n_{0}\right) \\
& =0 \text { for a.e. } \lambda \in \Omega .
\end{aligned}
$$

Taking $m=n_{0}+1$ in (3.15) yields

$$
\begin{aligned}
& M_{+}\left(\lambda+i 0, n_{0}\right) M_{-}\left(\lambda+i 0, n_{0}\right)-\overline{M_{+}\left(\lambda+i 0, n_{0}\right) M_{-}\left(\lambda+i 0, n_{0}\right)}=0 \\
& =\operatorname{Re}\left[M_{+}\left(\lambda+i 0, n_{0}\right)\right]\left\{\operatorname{Im}\left[M_{+}\left(\lambda+i 0, n_{0}\right)\right]-\operatorname{Im}\left[M_{-}\left(\lambda+i 0, n_{0}\right)\right]\right\} \\
& \quad \text { for a.e. } \lambda \in \Omega,
\end{aligned}
$$

since $s\left(\lambda, n_{0}+1, n_{0}\right)=1$. Taking $m=n_{1}$ in (3.15) finally proves

$$
\operatorname{Im}\left[M_{+}\left(\lambda+i 0, n_{0}\right)\right]=\operatorname{Im}\left[M_{-}\left(\lambda+i 0, n_{0}\right)\right] \quad \text { for a.e. } \lambda \in \Omega,
$$

and hence (iii) since $c\left(\lambda, n_{1}, n_{0}\right) s\left(\lambda, n_{1}, n_{0}\right) \neq 0$ for a.e. $\lambda \in \mathbb{R}$. (iii) implies (i) by combining (2.3), (3.12), and the real-valuedness of $c\left(\lambda, n, n_{0}\right)$ and $s\left(\lambda, n, n_{0}\right)$ for $\lambda \in \mathbb{R}$.

Next we turn to Dirichlet eigenvalues associated with $\tau$ corresponding to a Dirichlet boundary condition at $n \in \mathbb{Z}$. Associated with each spectral gap $\rho_{j}$ we set

$$
\mu_{j}(n)=\sup \left\{\left\{E_{2 j-1}\right\} \cup\left\{\lambda \in \rho_{j} \mid g(\lambda, n)<0\right\}\right\} \in \overline{\rho_{j}}, \quad j \in J .
$$

The strict monotonicity of $g(\lambda, n)$ with respect to $\lambda \in \rho_{j}$, that is,

$$
\frac{d}{d \lambda} g(\lambda, n)=\sum_{m \in \mathbb{Z}} G(\lambda, n, m)^{2}>0, \quad \lambda \in \rho_{j}
$$

then yields

$$
\begin{aligned}
& g(\lambda, n)<0, \quad \lambda \in\left(E_{2 j-1}, \mu_{j}(n)\right), \quad j \in J \\
& g(\lambda, n)>0, \quad \lambda \in\left(\mu_{j}(n), E_{2 j}\right),
\end{aligned}
$$

A more detailed analysis of the exponential Herglotz representation (3.1) of $g(z, n)$ then yields

Lemma 3.4. Assume (H.3.1) (i). Then

$$
\begin{aligned}
g(z, n) & =|g(i, n)| \exp \left\{\int_{\mathbb{R}}\left[\frac{1}{\lambda-z}-\frac{\lambda}{1+\lambda^{2}}\right] \xi(\lambda, n) d \lambda\right\} \\
& =\frac{-1}{z-E_{\infty}} \exp \left\{\int_{E_{0}}^{E_{\infty}} \frac{\xi(\lambda, n) d \lambda}{\lambda-z}\right\} \\
& =\frac{-1}{\left(z-E_{0}\right)^{1 / 2}\left(z-E_{\infty}\right)^{1 / 2}} \prod_{j \in J}\left[\frac{z-\mu_{j}(n)}{\left(z-E_{2 j-1}\right)^{1 / 2}\left(z-E_{2 j}\right)^{1 / 2}}\right],
\end{aligned}
$$


where the square root branch used is defined by

$$
z^{1 / 2}=\left|z^{1 / 2}\right| \exp [i \arg (z) / 2], \quad-\pi<\arg (z) \leqq \pi .
$$

In particular, denoting by $\chi_{\Omega}(\cdot)$ the characteristic function of the set $\Omega \subset \mathbb{R}$, one can represent $\xi(\lambda, n)$ by

$$
\begin{aligned}
\xi(\lambda, n)= & \frac{1}{2}\left[\chi_{\left(E_{0}, \infty\right)}(\lambda)+\chi_{\left(E_{\infty}, \infty\right)}(\lambda)\right] \\
& +\frac{1}{2} \sum_{j \in J}\left[\chi_{\left(E_{2 j-1}, \infty\right)}(\lambda)+\chi_{\left(E_{2 \jmath}, \infty\right)}(\lambda)-2 \chi_{\left(\mu_{j}(n), \infty\right)}(\lambda)\right] \\
= & \frac{1}{2} \chi_{\left(E_{0}, E_{\infty}\right)}(\lambda)+\frac{1}{2} \sum_{j \in J}\left[\chi_{\left(E_{2 j-1}, \mu_{j}(n)\right)}(\lambda)-\chi_{\left(\mu_{j}(n), E_{2 j}\right)}(\lambda)\right] \\
& +\chi_{\left(E_{\infty}, \infty\right)}(\lambda) \text { for a.e. } \lambda \in \mathbb{R} .
\end{aligned}
$$

For a later purpose we observe that the Laurent expression of $g(z, n)$ near $\frac{1}{z}=0$,

$$
g(z, n)=-\frac{1}{z}-\frac{b(n)}{z^{2}}+0\left(z^{-3}\right),
$$

combined with (3.21) implies the trace formula (cf. [17])

$$
b(n)=\frac{1}{2}\left(E_{0}+E_{\infty}\right)+\frac{1}{2} \sum_{j \in J}\left[E_{2 j-1}+E_{2 j}-2 \mu_{j}(n)\right] .
$$

Next, we denote for all $n \in \mathbb{Z}$,

$$
\begin{aligned}
\gamma_{ \pm, j}(n) & =-\lim _{\varepsilon \downarrow 0} i \varepsilon M_{ \pm}\left(\mu_{j}(n)+i \varepsilon, n\right) \geqq 0, \quad j \in J, \\
\gamma_{j}(n) & =\lim _{\varepsilon \downarrow 0} i \varepsilon g\left(\mu_{j}(n)+i \varepsilon, n\right)^{-1}=\gamma_{+, j}(n)+\gamma_{-, j}(n) \geqq 0, \quad j \in J, \\
\tilde{\gamma}_{ \pm, j}(n) & =-\lim _{\varepsilon \downarrow 0} i \varepsilon M_{ \pm}\left(\tilde{E}_{j}+i \varepsilon, n\right) \geqq 0, \quad j \in \tilde{J}, \\
\tilde{\gamma}_{j}(n) & =\lim _{\varepsilon \downarrow 0} i \varepsilon g\left(\tilde{E}_{j}+i \varepsilon, n\right)^{-1}=\tilde{\gamma}_{+, j}(n)+\tilde{\gamma}_{-, j}(n) \geqq 0, \quad j \in \tilde{J},
\end{aligned}
$$

where we used the fact that by the Herglotz property of $M_{ \pm}(z, n),-g(z, n)^{-1}$, the limits in (3.26)-(3.29) exist and take on nonnegative values (cf. (3.38) and (3.39). Associated with the limits (3.26)-(3.29) are the following ones $(n \in \mathbb{Z})$,

$$
\begin{aligned}
& \sigma_{j}(n)=\left\{\begin{array}{ll}
\lim _{\varepsilon \downarrow 0} h\left(\mu_{j}(n)+i \varepsilon, n\right)=\frac{\gamma_{+, j}(n)-\gamma_{-, j}(n)}{\gamma_{j}(n)} \in[-1,1] & \text { if } \gamma_{j}(n)>0 \\
2 & \text { if } \gamma_{j}(n)=0
\end{array},\right. \\
& \tilde{\sigma}_{j}(n)=\left\{\begin{array}{ll}
\lim _{\varepsilon \downarrow 0} h\left(\tilde{E}_{j}+i \varepsilon, n\right)=\frac{\tilde{\gamma}_{+, j}(n)-\tilde{\gamma}_{-, j}(n)}{\tilde{\gamma}_{j}(n)} \in[-1,1] & \text { if } \tilde{\gamma}_{j}(n)>0 \\
2 & \text { if } \tilde{\gamma}_{j}(n)=0
\end{array} .\right.
\end{aligned}
$$

The actual value of $\sigma_{j}(n)$ (resp. $\left.\tilde{\sigma}_{j}(n)\right)$ if $\gamma_{j}(n)=0$ (resp. $\left.\tilde{\gamma}_{j}(n)=0\right)$ in (3.30) (resp. (3.31) is immaterial. For notational convenience later on, we chose a value outside the interval $[-1,1]$ in this case.

We note that

$$
\mu_{j}(n) \notin \mathscr{A} \text { implies } \gamma_{j}(n)>0 .
$$


Finally, we summarize the direct spectral problem in the following

Theorem 3.5. Assume (H.3.1) and let $n \in \mathbb{Z}$. Then

(i)

$$
\begin{aligned}
\sigma_{p}\left(H_{ \pm, n}\right) & =\left\{\mu_{j}(n) \in \overline{\rho_{j}} \mid \sigma_{j}(n) \in_{[-1,1)}^{(-1,1]}\right\}_{j \in J} \\
& \cup\left\{\tilde{E}_{j} \in \mathscr{A} \mid \tilde{\sigma}_{j}(n) \in_{[-1,1)}^{(-1,1]}\right\}_{j \in \tilde{J}}
\end{aligned}
$$

(ii) If $\mu_{j}(n) \in \sigma_{p}(H)$ and $\stackrel{\left(\sim \tilde{\gamma}^{)}\right.}{{ }_{+, j}}(n)>0\left(\right.$ resp. $\left.\stackrel{(\sim)}{\gamma}_{-, j}(n)>0\right)$ then $\stackrel{(\sim)}{\gamma}_{-, j}(n)>0$ (resp. $\left.\tilde{\gamma}_{+, j}(n)>0\right)$, that is, if $\mu_{j}(n) \in \sigma_{p}(H)$ then $\mu_{j}(n) \in \sigma_{p}\left(H_{+, n}\right)$ if and only if $\mu_{j}(n) \in \sigma_{p}\left(H_{-, n}\right)$.

(iii) The following cases may occur:

$$
\begin{aligned}
& \mu_{j}(n) \in \rho_{j} \text { and } \sigma_{j}(n) \in\{-1,1\}, \\
& \mu_{j}(n) \in \sigma_{p}(H) \text { and } \sigma_{j}(n) \in[-1,1] \text { implying that } \mu_{j}(n) \in \sigma_{p}\left(H_{ \pm, n}\right), \\
& \mu_{j}(n)=\tilde{E}_{k} \text { and } \sigma_{j}(n)=\tilde{\sigma}_{k}(n) .
\end{aligned}
$$

Moreover, we have

$$
\begin{aligned}
& {\stackrel{(\sim)}{\sigma_{j}}}_{j}(n) \in[-1,1] \quad \text { if } \lim _{\varepsilon \downarrow 0} i \varepsilon g\left({\stackrel{(\sim)}{E_{j}}}_{j}+i \varepsilon, n\right)^{-1}>0, \\
& \stackrel{\left(\tilde{\sigma}_{j}^{)}\right.}{(n)}=2 \quad \text { if } \lim _{\varepsilon \downarrow 0} i \varepsilon g\left({\stackrel{(\sim)}{E_{j}}}_{j}+i \varepsilon, n\right)^{-1}=0 .
\end{aligned}
$$

(iv)

$$
\begin{aligned}
& \sigma_{a c}(H)=\sigma_{a c}\left(H_{ \pm, n}\right) \\
& =\overline{\left\{\lambda \in\left[E_{0}, E_{\infty}\right] \mid \xi\left(\lambda, n_{0}\right)=1 / 2\right\}^{\mathrm{ess}}} \text { for some } n_{0} \in \mathbb{Z} \\
& =\bigcup_{\substack{j \in J \\
E_{2 J} \neq E_{2 J}^{(r)}}} \Sigma_{j}, \\
& \sigma_{s c}(H)=\sigma_{s c}\left(H_{ \pm, n}\right)=\emptyset \text {, }
\end{aligned}
$$

$\sigma_{a c}(H)$ being of uniform spectral multiplicity two whereas $\sigma_{p}(H), \sigma_{p}\left(H_{ \pm}, n\right)$, and $\sigma_{a c}\left(H_{ \pm, n}\right)$ are all simple. In addition, if $d v_{ \pm, n}$ denote the measures associated with the Herglotz representations of $M_{ \pm}(z, n)$, that is,

$$
M_{+}(z, n)=\int_{\mathbb{R}} \frac{d v_{+, n}(\lambda)}{\lambda-z}, \quad M_{-}(z, n)=z-b(n)+\int_{\mathbb{R}} \frac{d v_{-, n}(\lambda)}{\lambda-z},
$$

then

$$
d v_{+, n, a c}=d v_{-, n, a c}, \quad d v_{ \pm, n, s c}=\emptyset .
$$

$d v_{ \pm, n}$ are both supported on infinite sets. (Here $\sigma_{a c}(\cdot), \sigma_{s c}(\cdot)$ abbreviate absolutely and singularly continuous spectra, respectively, $d v=d v_{a c}+d v_{s c}+d v_{p p} d e-$ notes the usual Lebesgue decomposition of measures, and $\bar{A}^{\text {ess }}$ denotes the essential closure of $A \subset \mathbb{R}$ with respect to Lebesgue measure, i.e., $\bar{A}^{-\mathrm{ess}}=\{\lambda \in \mathbb{R} \mid m(A \cap$ $(\lambda-\varepsilon, \lambda+\varepsilon))>0$ for all $\varepsilon>0\}, m(\cdot)$ denoting the Lebesgue measure.) 
Proof. If $F(z)$ denotes a Herglotz function with representation

$$
F(z)=c z+d+\int_{\mathbb{R}}\left[\frac{1}{\lambda-z}-\frac{\lambda}{1+\lambda^{2}}\right] d \omega(\lambda), \quad c \geqq 0, d \in \mathbb{R},
$$

then

$$
\omega\left(\left\{\lambda_{0}\right\}\right)=-\lim _{\varepsilon \downarrow 0} i \varepsilon F\left(\lambda_{0}+i \varepsilon\right) \quad \text { for all } \lambda_{0} \in \mathbb{R}
$$

yields (i), taking into account (3.26)-(3.29).

In order to prove (ii) one can argue as follows. ${\stackrel{(\sim)}{\gamma_{+, j}}}_{(n)}>0$ implies $\mu_{j}(n) \in$ $\sigma_{p}\left(H_{+, n}\right)$ by $(2.16),(3.26)$, and (3.27) and $\stackrel{(\sim)}{\gamma}_{j}(n)>0$ yields

$$
\lim _{\varepsilon \downarrow 0} \varepsilon g\left(\mu_{j}(n)+i \varepsilon, n\right)=0
$$

by (3.27) and (3.29). Since $\mu_{j}(n) \in \sigma_{p}(H),(2.13),(2.15),(3.26)$, and (3.27) yield

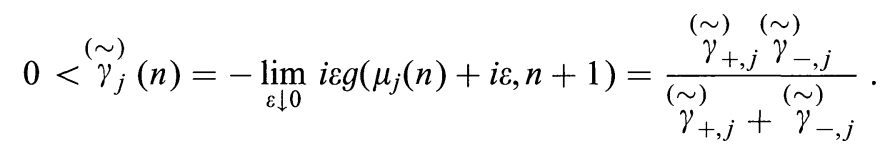

Hence ${ }^{\left({ }_{\gamma}\right)}{ }_{-, j}>0$ and thus $\mu_{j}(n) \in \sigma_{p}\left(H_{-, n}\right)$. Alternatively, one can invoke the eigenfunction $u_{+}\left(\mu_{j}(n), m\right)$ which then satisfies $u_{+}\left(\mu_{j}(n), n\right)=0, u_{+}\left(\mu_{j}(n), \cdot\right) \in l^{2}(\mathbb{Z})$ since $\mu_{j}(n) \in \sigma_{p}(H) \cap \sigma_{p}\left(H_{+, n}\right)$. The limit point property of $H$ at $\pm \infty$ then yields $u_{-}\left(\mu_{j}(n), \cdot\right)=C u_{+}\left(\mu_{j}(n), \cdot\right)$ for some constant $C$ and again one concludes that $\mu_{j}(n) \in \sigma_{p}\left(H_{-, n}\right)$.

(iii) is clear from (3.30) and (3.31).

Next, define

$$
\begin{aligned}
\Sigma_{ \pm, n, s c}= & \left\{\lambda \in\left[E_{0}, E_{\infty}\right] \mid \lim _{\varepsilon \downarrow 0} \operatorname{Im}\left[m_{ \pm}(\lambda+i \varepsilon, n)\right] \text { exists and equals }+\infty\right\}, \\
\Sigma_{s c}= & \left\{\lambda \in\left[E_{0}, E_{\infty}\right] \mid \lim _{\varepsilon \downarrow 0} \operatorname{Im}\left[g\left(\lambda+i \varepsilon, n_{0}\right)+g\left(\lambda+i \varepsilon, n_{0}+1\right)\right]\right. \\
& \text { exists and equals }+\infty\} \text { for some } n_{0} \in \mathbb{Z} .
\end{aligned}
$$

Then $\Sigma_{ \pm, n, s c}$ and $\Sigma_{s c}$ are minimal supports (cf., e.g., [4, 21, 22, 32, 33]) of $d v_{ \pm, n, s c}$ and $d v_{s c}^{t r}$, where $d v^{t r}=d v_{1,1}+d v_{2,2}$ abbreviates the trace measure of the $2 \times 2$ matrix-valued spectral measure $d v_{p, q}, 1 \leqq p, q \leqq 2$ of $H$ (derived from $g\left(z, n_{0}\right)$, $g\left(z, n_{0}+1\right), h\left(z, n_{0}\right)$, cf. (2.12)-(2.14)). By (2.12) one has

$$
-g(z, n)^{-1}=M_{+}(z, n)+M_{-}(z, n)
$$

and by the reflectionless property (3.8),

$$
-\lim _{\varepsilon \downarrow 0} g(\lambda+i \varepsilon, n)^{-1}=2 i \lim _{\varepsilon \downarrow 0} \operatorname{Im}\left[M_{ \pm}(\lambda+i \varepsilon, n)\right] \quad \text { for a.e. } \lambda \in \sigma_{e s s}(H) .
$$

Consider

$$
\sigma(H)^{0}=\left(\bigcup_{j \in J_{0}} \Sigma_{j}^{0}\right) \cup\left(\bigcup_{j \in \tilde{J}} \tilde{\Sigma}_{j}^{0}\right), \quad \Sigma_{j}^{0}=\left(E_{2 j}, E_{2 j}^{(r)}\right), \quad \tilde{\Sigma}_{j}^{0}=\left(\tilde{E}_{j}, \tilde{E}_{j}^{(r)}\right),
$$


where $A^{0}$ denotes the open interior of $A \subseteq \mathbb{R}$. Then the representation (3.21) shows that $\Sigma_{s c} \cap \sigma(H)^{0}=\emptyset$. But $\sigma(H) \backslash \sigma(H)^{0}$ is countable by Hypothesis (H.3.1) and hence $\sigma_{s c}(H)=\emptyset$. Equation (3.44) then also yields $\Sigma_{ \pm, s c} \cap \sigma(H)^{0}=\emptyset$ and $\sigma_{s c}\left(H_{ \pm, n}\right)=\emptyset, d v_{ \pm, n, s c}=0$, since $\sigma\left(H_{ \pm, n}\right) \backslash \sigma(H)^{0}$ is countable as well. Next, we recall that $\left(n_{0} \in \mathbb{Z}\right)$

$$
\begin{aligned}
\Sigma_{ \pm, a c} & =\left\{\lambda \in\left[E_{0}, E_{\infty}\right] \mid 0<\lim _{\varepsilon \downarrow 0} \operatorname{Im}\left[M_{ \pm}\left(\lambda+i \varepsilon, n_{0}\right)\right]<\infty \text { exists }\right\} \\
\Sigma_{a c} & =\left\{\lambda \in\left[E_{0}, E_{\infty}\right] \mid 0<\lim _{\varepsilon \downarrow 0} \operatorname{Im}\left[g\left(\lambda+i \varepsilon, n_{0}\right)\right]<\infty \text { exists }\right\}
\end{aligned}
$$

are minimal supports of $d v_{ \pm, n, a c}$ and $d v_{a c}^{t r}$, respectively. By (3.44) one infers $d v_{+, n, a c}=d v_{-, n, a c}$ and hence (3.37). Equation (3.34) then follows from (3.8), (3.44), (3.46), (3.47), and Theorem 5.2 of [17] which states

$$
\sigma_{a c}(H)=\overline{\left\{\lambda \in\left[E_{0}, E_{\infty}\right] \mid 0<\xi\left(\lambda, n_{0}\right)<1\right\}^{\mathrm{ess}}} .
$$

Finally, spectral multiplicity two on $\sigma_{a c}(H)$ is a consequence of (3.8) and (3.44); $\sigma_{p}(H)$ is simple since $H$ is in the limit point case at $\pm \infty$, and half-line spectra $\sigma\left(H_{ \pm, n}\right)$ are well-known to be simple. $d v_{ \pm, n}$ are both supported on infinite sets since $H_{ \pm, n}$ are defined on the discrete half-lines $\mathbb{Z} \cap(0, \pm \infty)$.

That $H, H_{ \pm, n}$ have purely absolutely continuous spectra on $\sigma(H)^{0}$ (cf. (3.45) also follows from Theorem 3.1 in [27].

\section{The Inverse Spectral Problem}

In this section we describe our principal new result on the isospectral set of selfadjoint Jacobi operators satisfying Hypothesis (H.3.1).

We start by introducing the following hypothesis.

Hypothesis 4.1. (i) Let

$$
\Sigma=\mathbb{R} \backslash \bigcup_{j \in J_{0} \cup\{\infty\}} \rho_{j},
$$

where $J \subseteq \mathbb{N}, J_{0}=J \cup\{0\}$,

$$
\begin{gathered}
\rho_{0}=\left(-\infty, E_{0}\right), \quad \rho_{\infty}=\left(E_{\infty}, \infty\right), \\
\rho_{j}=\left(E_{2 j-1}, E_{2 j}\right), \quad E_{0} \leqq E_{2 j-1}<E_{2 j} \leqq E_{\infty}, \quad j \in J, \\
-\infty<E_{0}<E_{\infty}<\infty, \quad \rho_{j} \cap \rho_{k}=\emptyset \text { for } j \neq k .
\end{gathered}
$$

By $\Sigma_{d}$ we denote the set of isolated (discrete) points of $\Sigma$.

(ii) The set $\mathscr{A}$ of all accumulation points of the set $\left\{E_{2 j-1}, E_{2 j}\right\}_{j \in J}$ is assumed to be countable and denoted by

$$
\mathscr{A}=\left\{\tilde{E}_{j}\right\}_{j \in \tilde{J}}, \quad \tilde{J} \subseteq \mathbb{N} .
$$

(iii) We introduce the set $\left\{\mu_{j} \in \overline{\rho_{j}}\right\}_{j \in J}$ and define $g(z)$ as in (3.21). In addition, we introduce

$$
\left\{\left(\mu_{j}, \sigma_{j}\right) \in \overline{\rho_{j}} \times[-1,1]\right\}_{j \in J},
$$


where

$$
\begin{gathered}
\sigma_{j} \in\{-1,1\} \quad \text { if } \mu_{j} \in \rho_{j}, \\
\sigma_{j} \in(-1,1) \quad \text { if } \mu_{j} \in \partial \rho_{j} \cap \Sigma_{d} . \text { In this case } \\
\mu_{j}=\mu_{k} \text { and } \sigma_{j}=\sigma_{k} \text { for some } j \neq k \in J .
\end{gathered}
$$

Next, we consider

$$
\left\{\left(\tilde{E}_{j}, \tilde{\sigma}_{j}\right) \in \mathscr{A} \times\{[-1,1] \cup\{2\}\}\right\}_{j \in \tilde{J}},
$$

where

$$
\begin{aligned}
& \tilde{\sigma}_{j} \in[-1,1] \quad \text { if } \lim _{\varepsilon \downarrow 0} i \varepsilon g\left(\tilde{E}_{j}+i \varepsilon\right)^{-1}>0, \\
& \tilde{\sigma}_{j}=2 \text { if } \lim _{\varepsilon \downarrow 0} i \varepsilon g\left(\tilde{E}_{j}+i \varepsilon\right)^{-1}=0 .
\end{aligned}
$$

Finally,

$$
\text { if } \mu_{j}=\tilde{E}_{k} \text { for some } j \in J, k \in \tilde{J} \text {, then } \sigma_{j}=\tilde{\sigma}_{k} \text {. }
$$

(iv) If $\Sigma=\overline{\Sigma_{d}}$, the index sets

$$
J_{ \pm}=\left\{j \in J \mid \sigma_{j} \in_{[-1,1)}^{(-1,1]}\right\} \text { are infinite . }
$$

Remark 4.2. Conditions (i) and (ii) just reintroduce the necessary notation from Hypothesis (H.3.1). Equations (4.4)-(4.8) in condition (iii) take care of items (ii) and (iii) in Theorem 3.5. In particular, the fact that two Dirichlet eigenvalues must simultaneously hit a point in $\sigma_{d}(H)$ is taken into account in (4.6). If only a single Dirichlet eigenvalue $\mu_{j}$ would hit a point $E^{*} \in \sigma_{d}(H)$, then, since $E^{*}$ necessarily occurs twice in the product $(3.21)$, the term $\left[z-\mu_{j}\right] /\left[\left(z-E^{*}\right)^{1 / 2}\left(z-E^{*}\right)^{1 / 2}\right]$ simply drops out and one would have "lost" $E^{*}$. In other words, such a deformation of $\mu_{j}(n)$ would be nonisospectral. (A detailed account of such (non)isospectral deformations will appear in [19].) Condition (4.9) is a consistency requirement and condition (iv) reflects the fact that we are working with infinite matrix operators on the discrete half-lines $\mathbb{Z} \cap(0, \pm \infty)$.

Given Hypothesis (H.4.1) we define the set of Dirichlet and accumulation data

$$
\mathscr{D}_{\Sigma}=\left\{\left\{\left(\mu_{j}, \sigma_{j}\right) \in \overline{\rho_{J}} \times[-1,1]\right\}_{j \in J},\left\{\tilde{\sigma}_{j}\right\}_{j \in \tilde{J}} \mid \text { assuming (H.4.1) }\right\} .
$$

The isospectral set of self-adjoint reflectionless Jacobi operators $H$ satisfying (H.3.1) with $\sigma(H)=\Sigma$ is denoted by

$$
I(\Sigma)=\left\{\text { Jacobi operators } H \text { in } l^{2}(\mathbb{Z}) \mid \sigma(H)=\Sigma\right\} .
$$

Theorem 4.3. Suppose $\Sigma$ satisfies (H.4.1). Then the map

$$
\left\{\begin{array}{l}
I(\Sigma) \rightarrow \mathscr{D}_{\Sigma} \\
H \rightarrow\left\{\left\{\left(\mu_{j}^{\circ}, \sigma_{j}^{\circ}\right)\right\}_{j \in J},\left\{\tilde{\sigma}_{j}^{\circ}\right\}_{j \in \tilde{J}}\right\},
\end{array}\right.
$$

constructed in Theorem 3.5 is a bijection, where

$$
\begin{aligned}
\sigma(H)= & \Sigma, \\
\sigma_{p}\left(H_{ \pm, n_{0}}\right)=\left\{\mu_{j}^{\circ} \in \overline{\rho_{j}} \mid \sigma_{j}^{\circ} \in_{[-1,1)}^{(-1,1]}\right\}_{j \in J} \cup\left\{\tilde{E}_{j} \in \mathscr{A} \mid \tilde{\sigma}_{j}^{\circ} \in_{[-1,1)}^{(-1,1]}\right\}_{j \in \tilde{J}} & \\
& \text { for some } n_{0} \in \mathbb{Z} .
\end{aligned}
$$


Proof. We first show that the map (4.13) is surjective. Fix a point

$$
\left\{\left\{\left(\mu_{j}^{\circ}, \sigma_{j}^{\circ}\right)\right\}_{j \in J},\left\{\tilde{\sigma}_{j}^{\circ}\right\}_{j \in \tilde{J}}\right\} \in \mathscr{D}_{\Sigma} .
$$

We shall construct a unique Jacobi operator $H \in I(\Sigma)$ satisfying (4.14) and (4.15). Given (4.16), define $g\left(z, n_{0}\right)$ as in (3.21). Let $v_{n_{0}}$ be the measure in the Herglotz representation of $-g\left(z, n_{0}\right)^{-1}$, that is

$$
-g\left(z, n_{0}\right)^{-1}=z-b\left(n_{0}\right)+\int_{\mathbb{R}} \frac{d v_{n_{0}}(\lambda)}{\lambda-z},
$$

with

$$
b\left(n_{0}\right)=\frac{1}{2}\left(E_{0}+E_{\infty}\right)+\frac{1}{2} \sum_{j \in J}\left[E_{2 j-1}+E_{2 j}-2 \mu_{j}^{\circ}\right] .
$$

Next, we split up $v_{n_{0}}=v_{+, n_{0}}+v_{-, n_{0}}$ as follows. Since the pure point part of $v_{n_{0}}$ is supported on $\left\{\mu_{j}^{\circ} \in \overline{\rho_{j}}\right\}$ we define

$$
v_{ \pm, n_{0}}\left(\left\{\mu_{j}^{\circ}\right\}\right)=\frac{1}{2}\left(1 \pm \sigma_{j}^{\circ}\right) v_{n_{0}}\left(\left\{\mu_{j}^{\circ}\right\}\right)
$$

and similarly,

$$
v_{ \pm, n_{0}}\left(\left\{\tilde{E}_{j}\right\}\right)=\frac{1}{2}\left(1 \pm \tilde{\sigma}_{j}^{\circ}\right) v_{n_{0}}\left(\left\{\tilde{E}_{j}\right\}\right) .
$$

(The split up of the pure point part in (4.19) resembles the one in Theorem 3.6 of [18] in the case of Schrödinger operators with purely discrete spectra.) The absolutely continuous part of $v_{n_{0}}$ is then split up according to Lemma 3.3, respectively (3.44), by

$$
v_{ \pm, n_{0}, a c}=\frac{1}{2} v_{n_{0}, a c}
$$

We note that

$$
v_{n_{0}, s c}=v_{ \pm, n_{0}, s c}=0
$$

by the argument following (3.45). Next, define

$$
a\left(n_{0}\right)=\left[\int_{\mathbb{R}} d v_{+, n_{0}}(\lambda)\right]^{1 / 2}, \quad a\left(n_{0}-1\right)=\left[\int_{\mathbb{R}} d v_{-, n_{0}}(\lambda)\right]^{1 / 2},
$$

and consider the probability measures

$$
\omega_{+, n_{0}}=a\left(n_{0}\right)^{-2} v_{+, n_{0}}, \quad \omega_{-, n_{0}}=a\left(n_{0}-1\right)^{-2} v_{-, n_{0}}
$$

(which are both supported on infinite sets). $\omega_{ \pm, n_{0}}$ enable one to compute $H_{ \pm, n_{0}}$ by the moment approach as outlined, for instance, in [1], Ch. 4 and [7], Ch. 7. One obtains,

$$
\begin{gathered}
a(n)=\int_{\mathbb{R}} \lambda s_{ \pm}\left(\lambda, n, n_{0}\right) s_{ \pm}\left(\lambda, n+1, n_{0}\right) d \omega_{ \pm, n_{0}}(\lambda), \quad \pm\left(n-n_{0}\right) \geqq\left\{\begin{array}{l}
1 \\
2
\end{array},\right. \\
b(n)=\int_{\mathbb{R}} \lambda s_{ \pm}\left(\lambda, n, n_{0}\right)^{2} d \omega_{ \pm, n_{0}}(\lambda), \quad \pm\left(n-n_{0}\right) \geqq 1
\end{gathered}
$$


where $s_{ \pm}\left(\lambda, n, n_{0}\right), \pm\left(n-n_{0}\right) \geqq 1$ are polynomials (of degree $\left.\pm\left(n-n_{0}\right)\right)$ orthonormal with respect to $d \omega_{ \pm, n_{0}}(\lambda)$. This determines $H$ and (4.15). Introducing

$$
\begin{gathered}
M_{+}\left(z, n_{0}\right)=a\left(n_{0}\right)^{2} \int_{\mathbb{R}} \frac{d \omega_{+, n_{0}}(\lambda)}{\lambda-z}, \\
M_{-}\left(z, n_{0}\right)=z-b\left(n_{0}\right)+a\left(n_{0}-1\right)^{2} \int_{\mathbb{R}} \frac{d \omega_{-, n_{0}}(\lambda)}{\lambda-z},
\end{gathered}
$$

one verifies (4.14) using (2.12), (2.14), and (2.15). It remains to show that the map (4.13) is injective. Suppose $H_{1} \in I(\Sigma)$ and $H_{2} \in I(\Sigma)$ are both mapped to the same point in (4.16). Then one infers $v_{ \pm, 1, n_{0}}=v_{ \pm, 2, n_{0}}$ and $b_{1}\left(n_{0}\right)=b_{2}\left(n_{0}\right)$ (where, in obvious notation, $v_{ \pm, j, n_{0}}$ and $b_{j}$ refer to $\left.H_{j}, j=1,2\right)$ and hence $H_{1}=H_{2}$.

We conclude with a simple example illustrating an explicit construction to the effect that an accumulation point of eigenvalues of $H$ may or may not be an eigenvalue of $H$.

Example 4.4. Suppose $H$ satisfies (H.3.1), $H$ has pure point spectrum only, and $\mathscr{A} \neq \emptyset$. Let $\tilde{E}_{j_{0}} \in \mathscr{A}$ and define

$$
\tilde{\gamma}_{j_{0}}=\lim _{\varepsilon \downarrow 0} i \varepsilon g\left(\tilde{E}_{j_{0}}+i \varepsilon, 0\right)^{-1}
$$

and

$$
g_{\delta}(z, 0)=-\left[-g(z, 0)^{-1}-\left(\delta-\tilde{\gamma}_{j_{0}}\right)\left(z-\tilde{E}_{j_{0}}\right)^{-1}\right]^{-1}, \quad \delta \geqq 0 .
$$

Then $\tilde{\gamma} \geqq 0$ and $g_{\delta}$ is a Herglotz function corresponding to a pure point measure in its representation of the type (3.38). Computing the zeros $\mu_{\delta, j}$ of $g_{\delta}(z, 0)$ and choosing $\sigma_{\delta, j}, \tilde{\sigma}_{\delta, j} \in[-1,1] \times\{2\}$ according to (H.4.1) yields a corresponding Jacobi operator $H_{\delta}$ by Theorem 4.8. Since

$$
\lim _{\varepsilon \downarrow 0} i \varepsilon g_{\delta}\left(\tilde{E}_{j_{0}}+i \varepsilon, 0\right)^{-1}=\delta
$$

one obtains the following case distinctions:

(i) $\delta=0$, then $\tilde{E}_{j_{0}} \notin \sigma_{p}\left(H_{\delta, \pm}\right)$.

(ii) $\delta>0, \tilde{\sigma}_{\delta, j_{0}} \in\{ \pm 1\}$, then $\tilde{E}_{j_{0}} \in \sigma_{p}\left(H_{\delta, \tilde{\sigma}_{\delta, j_{0}}}\right), \tilde{E}_{j_{0}} \notin \sigma_{p}\left(H_{\delta}\right)$.

(iii) $\delta>0, \tilde{\sigma}_{\delta_{,} j_{0}} \in(-1,1)$, then $\tilde{E}_{j_{0}} \in \sigma_{p}\left(H_{\delta, \pm}\right) \cap \sigma_{p}\left(H_{\delta}\right)$.

Case (i) is clear (in this case $\tilde{E}_{j_{0}}$ may or may not belong to $\sigma_{p}(H)$ ). Case (ii) follows from Theorem 3.5 (ii). In case (iii) one has $\lim _{\varepsilon \downarrow 0} \varepsilon g_{\delta}\left(\tilde{E}_{j_{0}}+i \varepsilon, 0\right)=0$, but $-\lim _{\varepsilon \downarrow 0} i \varepsilon g_{\delta}\left(\tilde{E}_{j_{0}}+i \varepsilon, 1\right)>0$ in analogy to $(3.40) . \tilde{E}_{j_{0}} \in \sigma_{p}\left(H_{\delta}\right)$ then follows from (2.15).

Acknowledgements. F.G. would like to thank Barry Simon for numerous discussions and joint work on inverse spectral problems which helped to shore up the foundations for this paper. M.K. wishes to thank Walter Craig for discussions and the Departments of Mathematics at Brown University and the University of Missouri-Columbia for an invitation which made this work possible. 


\section{References}

1. Akhiezer, N.I.: The Classical Moment Problem. Edinburgh: Oliver and Boyd, 1965

2. Antony, A.J., Krishna, M.: Almost periodicity of some Jacobi matrices. Proc. Indian Acad. Sci. (Math. Sci.) 102, 175-188 (1992)

3. Antony, A.J., Krishna, M.: Inverse spectral theory for Jacobi matrices and their almost periodicity. Proc. Indian Acad. Sci. (Math. Sci.) 104, 777-818 (1994)

4. Aronszajn, N.: On a problem of Weyl in the theory of singular Sturm-Liouville equations. Am. J. Math. 79, 597-610 (1957)

5. Aronszajn, N., Donoghue, W.F.: On exponential representations of analytic functions in the upper half-plane with positive imaginary part. J. Anal. Math. 5, 321-388 (1956-57)

6. Belokolos, E.D., Bobenko, A.I., Enol'skii, V.Z., Its, A.R., Matveev, V.B.: Algebro-Geometric Approach to Nonlinear Integrable Equations. Berlin: Springer, 1994

7. Berezanski1̌, Ju.M.: "Expansions in Eigenfunctions of Self-Adjoint Operators". Providence, R.I.: Am. Math. Soc., 1968

8. Bulla, W., Gesztesy, F., Holden, H., Teschl, G.: Algebro-geometric quasi-periodic finite-gap solutions of the Toda and Kac-van Moerbeke hierarchy. Memoirs Amer. Math. Soc., to appear

9. Carmona, R., Kotani, S.: Inverse spectral theory for random Jacobi matrices. J. Stat. Phys. 46, 1091-1114 (1987)

10. Carmona, R., Lacroix, J.: Spectral Theory of Random Schrödinger Operators. Boston: Birkhäuser, 1990

11. Craig, W.: The trace formula for Schrödinger operators on the line. Commun. Math. Phys. 126, 379-407 (1989)

12. Date, E., Tanaka, S.: Analogue of inverse scattering theory for the discrete Hill's equation and exact solutions for the periodic Toda lattice. Progr. Theoret. Phys. 56, 457-465 (1976)

13. Davies, E.B., Simon, B.: Scattering theory for systems with different spatial asymptotics on the left and right. Commun. Math. Phys. 63, 277-301 (1978)

14. Deift, P., Simon, B.: Almost periodic Schrödinger operators III. The absolutely continuous spectrum in one dimension. Commun. Math. Phys. 90, 389-411 (1983)

15. Dubrovin, B.A., Matveev, V.B., Novikov, S.P.: Non-linear equations of Korteweg-De Vries type, finite-zone linear operators, and Abelian varieties. Russ. Math. Surv. 31:1, 59-146 (1976)

16. Gesztesy, F., Nowell, R., Pötz, W.: One-dimensional scattering theory for quantum systems with nontrivial spatial asymptotics. Adv. Diff. Eqs., to appear

17. Gesztesy, F., Simon, B.: The xi function, Acta Math. 176, 49-71 (1996)

18. Gesztesy, F., Simon, B.: Uniqueness theorems in inverse spectral theory for one-dimensional Schrödinger operators. Trans. Am. Math. Soc. 348, 349-373 (1996)

19. Gesztesy, F., Simon, B., Teschl, G.: Spectral deformations of one-dimensional Schrödinger operators. J. Anal. Math., to appear

20. Gesztesy, F., Teschl, G.: Commutation methods for Jacobi operators. J. Diff. Eqs. 128, 252299 (1996)

21. Gilbert, D.J.: On subordinacy and analysis of the spectrum of Schrödinger operators with two singular endpoints. Proc. Roy. Soc. Edinburgh 112A, 213-229 (1989)

22. Gilbert, D.J., Pearson, D.B.: On subordinacy and analysis of the spectrum of one-dimensional Schrödinger operators. J. Math. Anal. Appl. 128, 30-56 (1987)

23. Kac, M., van Moerbeke, P.: On some periodic Toda lattices. Proc. Nat. Acad. Sci. USA 72, 1627-1629 (1975)

24. Kac, M., van Moerbeke, P.: A complete solution of the periodic Toda problem. Proc. Nat. Acad. Sci. USA 72, 2879-2880 (1975)

25. Kotani, S., Krishna, M.: Almost periodicity of some random potentials. J. Funct. Anal. 78, 390-405 (1988)

26. Levitan, B.M.: Inverse Sturm-Liouville Problems. Utrecht: VNU Science Press, 1987

27. Mantlik, F., Schneider, A.: Note on the absolutely continuous spectrum of Sturm-Liouville operators. Math. Z. 205, 491-498 (1990)

28. McKean, H.P., van Moerbeke, P.: Hill and Toda curves. Commun. Pure Appl. Math. 33, 23-42 (1980)

29. van Moerbeke, P.: The spectrum of Jacobi matrices. Invent. Math. 37, 45-81 (1976) 
30. van Moerbeke, P.: About isospectral deformations of discrete Laplacians. In: "Global Analysis," M. Grmela and J.E. Marsden (eds.), Lecture Notes in Mathematics 755, Berlin: Springer, 1979, pp. 313-370

31. van Moerbeke, P., Mumford, D.: The spectrum of difference operators and algebraic curves. Acta Math. 145, 97-154 (1979)

32. Simon, B.: Spectral analysis of rank one perturbations and applications. In: "Mathematical Quantum Theory II: Schrödinger Operators,” J. Feldman, R. Froese, L.M. Rosen (eds.), CRM Proceedings and Lecture Notes Vol. 8, Providence, R.I.: Am. Math. Soc., 1995, pp. 109-149

33. Simon, B.: $L^{p}$ norms of the Borel transform and the decomposition of measures. Proc. Am. Math. Soc. 123, 3749-3755 (1995)

34. Sodin, M.L., Yuditskiĭ, P.M.: Infinite-zone Jacobi matrices with pseudo-extendible Weyl functions and homogeneous spectrum. Russ. Acad. Sci. Dokl. Math. 49, 364-368 (1994)

35. Sodin, M., Yuditskii, P.: Almost periodic Jacobi matrices with homogeneous spectrum, infinite dimensional Jacobi inversion, and Hardy spaces of character-automorphic functions. Preprint, 1994

36. Toda, M.: Theory of Nonlinear Lattices. $2^{\text {nd }}$ enl. ed., Berlin: Springer, 1989

Communicated by B. Simon 
